\title{
The role of sacral slope in lumbosacral fusion: a biomechanical study
}

\author{
Doniel Drazin, MD, ${ }^{1}$ Mir Hussain, BS, ${ }^{2}$ Jonathan Harris, MS, ${ }^{2}$ John Hao, BS, ${ }^{2}$ Matt Phillips, BS, ${ }^{2}$ \\ Terrence T. Kim, MD, ${ }^{3}$ J. Patrick Johnson, MD, ${ }^{1,4}$ and Brandon Bucklen, PhD ${ }^{2}$

\begin{abstract}
Departments of ${ }^{1}$ Neurosurgery and ${ }^{3}$ Orthopedics, Cedars-Sinai Medical Center, Los Angeles, California; ${ }^{2}$ Globus Medical, Inc., Valley Forge Business Center, Audubon, Pennsylvania; and ${ }^{4}$ Department of Neurosurgery, University of California, Davis,
\end{abstract} \\ Sacramento, California
}

\begin{abstract}
OBJECT Abnormal sacral slope (SS) has shown to increase progression of spondylolisthesis, yet there exists a paucity in biomechanical studies investigating its role in the correction of adult spinal deformity, its influence on lumbosacral shear, and its impact on the instrumentation selection process. This in vitro study investigates the effect of SS on 3 anterior lumbar interbody fusion constructs in a biomechanics laboratory.
\end{abstract}

METHODS Nine healthy, fresh-frozen, intact human lumbosacral vertebral segments were tested by applying a 550 $\mathrm{N}$ axial load to specimens with an initial SS of $20^{\circ}$ on an MTS Bionix test system. Testing was repeated as SS was increased to $50^{\circ}$, in $10^{\circ}$ increments, through an angulated testing fixture. Specimens were instrumented using a standalone integrated spacer with self-contained screws (SA), an interbody spacer with posterior pedicle screws (PPS), and an interbody spacer with anterior tension band plate (ATB) in a randomized order. Stiffness was calculated from the linear portion of the load-deformation curve. Ultimate strength was also recorded on the final construct of all specimens ( $n=3$ per construct) with SS of $40^{\circ}$.

RESULTS Axial stiffness (N/mm) of the L5-S1 motion segment was measured at various angles of SS: for SA 292.9 $\pm 142.8\left(20^{\circ}\right), 277.2 \pm 113.7\left(30^{\circ}\right), 237.0 \pm 108.7\left(40^{\circ}\right), 170.3 \pm 74.1\left(50^{\circ}\right)$; for PPS $371.2 \pm 237.5\left(20^{\circ}\right), 319.8 \pm 167.2$ $\left(30^{\circ}\right), 280.4 \pm 151.7\left(40^{\circ}\right), 233.0 \pm 117.6\left(50^{\circ}\right)$; and for ATB $323.9 \pm 210.4\left(20^{\circ}\right), 307.8 \pm 125.4\left(30^{\circ}\right), 249.4 \pm 126.7$ $\left(40^{\circ}\right), 217.7 \pm 99.4\left(50^{\circ}\right)$. Axial compression across the disc space decreased with increasing SS, indicating that SS beyond $40^{\circ}$ threshold shifted L5-S1 motion into pure shear, instead of compression-shear, defining a threshold. Trends in ultimate load and displacement differed from linear stiffness with SA > PPS > ATB.

CONCLUSIONS At larger SSs, bilateral pedicle screw constructs with spacers were the most stable; however, none of the constructs were significantly stiffer than intact segments. For load to failure, the integrated spacer performed the best; this may be due to angulations of integrated plate screws. Increasing SS significantly reduced stiffness, which indicates that surgeons need to consider using more aggressive fixation techniques.

http://thejns.org/doi/abs/10.3171/2015.3.SPINE14557

KEY WORDS sacral slope; integrated spacer; stand-alone; anterior interbody fusion; biomechanics; biomechanical study

$\mathrm{W}$ HILE innovations in spine surgery have afforded patients relief and independence, the field has yet to attain a reliable technique for solid arthrodesis of the lumbosacral junction. . $^{1,5,8,19,23,31}$ Long fusions to the sacrum, such as those necessary for treating osteoporotic patients with degenerative neuromuscular scoliosis and patients who have undergone spondylolisthesis reduction, have been particularly challenging. $5,8,23,36$ Clinical outcomes have confirmed various complications, which include failure of the bone-metal interface, fracture of the implants, loss of alignment, and pseudarthrosis. ${ }^{33}$ Low bone mineral density, load on the lumbosacral region, and joint angulation may affect fusion at this junction.

The level of stabilization required across the lumbosacral junction to prevent slippage after implantation is poorly understood. Traditional circumferential fusion techniques have evolved with advances in anterior implants including plating and stand-alone interbody grafts. ${ }^{7}$ The

ABBREVIATIONS ATB = anterior tension band plate with interbody spacer; PPS = posterior pedicle screws placed with interbody spacer; SA = stand-alone integrated spacer with self-contained screws; SS = sacral slope.

SUBMITTED July 25, 2014. ACCEPTED March 24, 2015.

INCLUDE WHEN CITING Published online August 14, 2015; DOI: 10.3171/2015.3.SPINE14557. 
efficacy of these stand-alone interbody grafts has called into question the need for both anterior plating and posterior fixation technique. ${ }^{43}$

Various studies have addressed the relationship between the position of the pelvis and spinal alignment. $13,30,38,40$ Through understanding this relationship in healthy subjects, parameters have been established for diagnosis and treatment in patients with spinal deformity. The pelvis in the sagittal plane is commonly analyzed using 3 angular measurements: pelvic tilt, pelvic incidence, and sacral slope. ${ }^{21}$ Sacral slope is defined as the angle between the horizontal and the sacral plate, and is a critical spinal parameter in the analysis of sagittal balance that has been correlated to the progression of spondylolisthesis. ${ }^{4,18}$ The higher the sacral slope, the greater the likelihood of spondylolisthesis. ${ }^{13,18}$ It is hypothesized that the increased sacral slope creates greater lumbosacral lordosis, which in turn creates higher stress across the pars region. ${ }^{27}$ The influence of sacral slope on lumbosacral shear and its importance in selecting implants has not been studied in a biomechanics laboratory.

Three most common surgical strategies for lumbosacral supplemental fixation are bilateral posterior pedicle screws placed with an interbody spacer (PPS), an anterior tension band plate with an interbody spacer (ATB), and an anterior stand-alone integrated spacer with self-contained screws, otherwise known as an integrated spacer and plate (SA). It is unclear which of these techniques is best suited for the spinopelvic geometrical and angular challenges of the lumbosacral spine. To the authors' knowledge, research has not assessed ATB fusion compared with SA or PPS constructs (Fig. 1) or how fixation is affected by sacral slope. Neither has research been performed for the resultant increase in shear on anterior versus the circumferential fusion constructs. The results of this research may be used to develop selection criteria for optimizing clinical outcomes at L5-S1.

\section{Methods}

\section{Specimen Preparation}

Nine healthy, fresh-frozen cadaver spines from L-5 to S-1 were used in this study. The medical history of each donor was reviewed along with radiographs to exclude any specimens with spinal trauma, malignancy, deformity, or fractures that would otherwise affect the outcome of the testing. Specimens were carefully dissected leaving only ligaments, bones, and intervertebral discs of the segments desired. Each specimen was potted at L-5 proximally and at S-1 distally using a 1:1 mixture of Bondo auto filler (Bondo MarHyde Corp.) and fiberglass resin (Home-Solution All Purpose, Bondo MarHyde Corp.). All specimens were double wrapped in plastics bags and stored at $-20^{\circ} \mathrm{C}$.

\section{Surgical Constructs}

Initially, each specimen was tested to develop a baseline value for axial stiffness. The intact value was established by compressively loading the specimens at $20^{\circ}-50^{\circ}$ sacral slope with increments of $10^{\circ}$. Axial stiffness was calculated by using the most linear portion of the load-displacement curve. Determination of sacral slope angles were de-

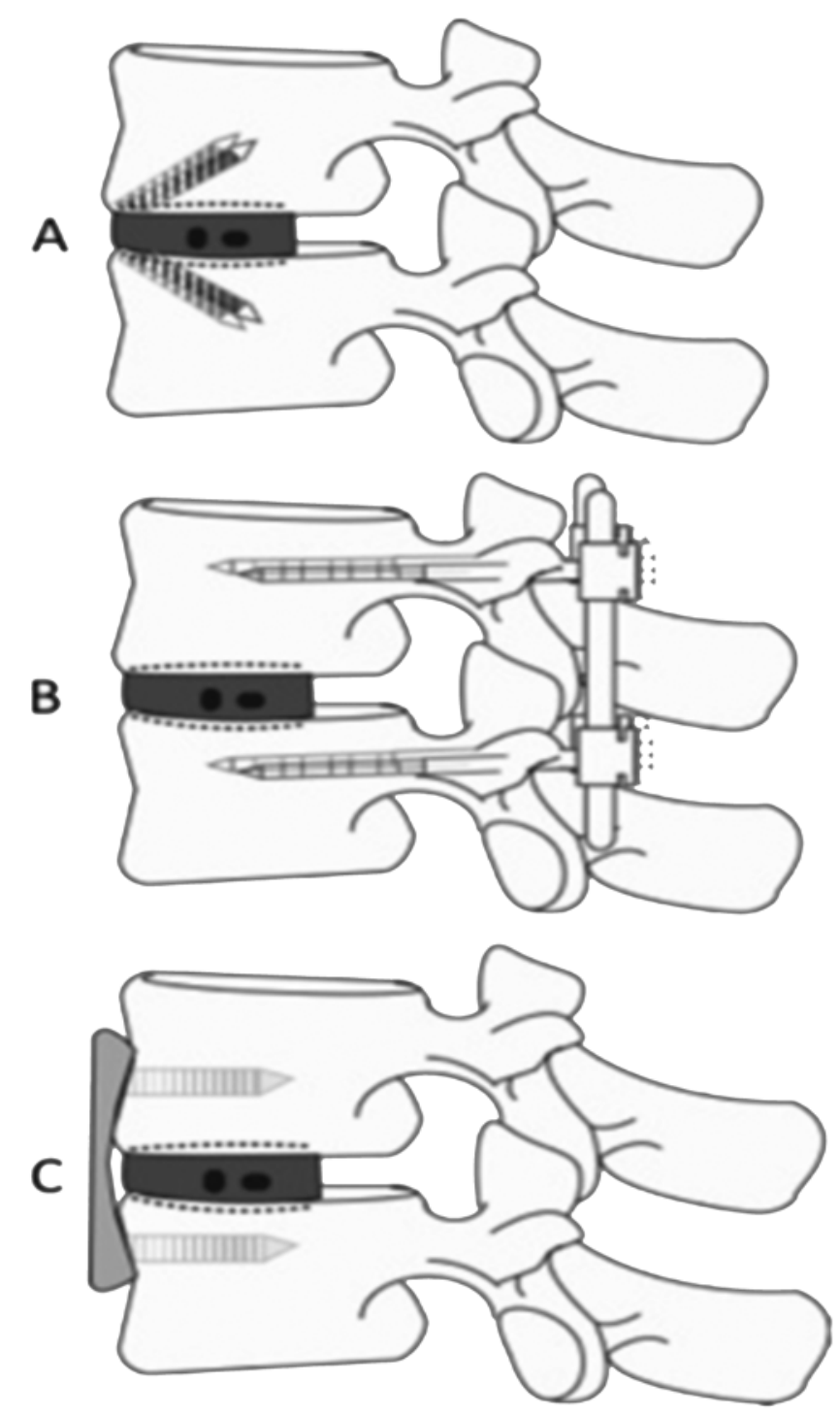

FIG. 1. Illustrations showing the 3 different fusion constructs assessed in the present study. A: Stand-alone anterior lumbar interbody spacer with integrated plate and screws. B: Anterior lumbar interbody spacer with posterior pedicle screws. C: Anterior lumbar interbody graft with anterior tension band plate and screws.

rived from values reported in previous literature. ${ }^{9,12,25}$ The $20^{\circ}-30^{\circ}$ range represent normal angles, and the $40^{\circ}-50^{\circ}$ range is considered to be high sacral slope angulation. After intact specimens were tested, a complete anterior lumbar discectomy was performed with removal of all disc material. Care was taken to preserve the bony endplates of L-5 and S-1. The posterior longitudinal ligament was also resected. After complete discectomy, the interbody implant was inserted with good interference fit ensuring the spacer was secure. Fluoroscopic imaging was used to place the interbodies, aiding precise placement throughout the constructs. SA constructs required insertion of 3 screws (2 into S-1 and 1 at L-5) through the integrated plate (INDEPENDENCE, Globus Medical Inc.). The L-5 screw is a $35^{\circ}$ cranial angle centered to the interbody while the S-1 screws are a $35^{\circ}$ caudal angle that diverges 


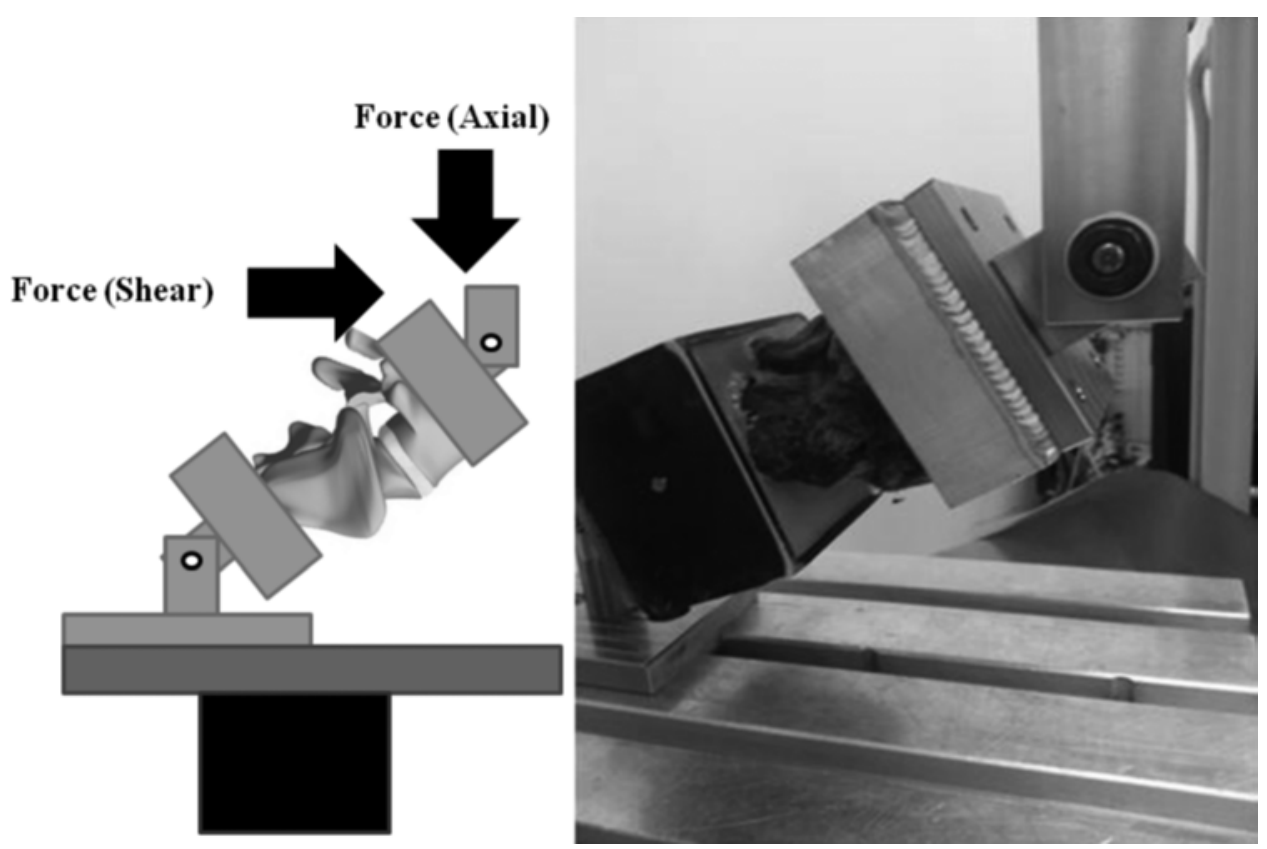

FIG. 2. Illustration showing the L5-S1 segment in the custom-built loading apparatus (left). Photograph showing the intact specimen during testing at $50^{\circ}$ with axial load applied (right).

$14^{\circ}$ per each screw. The SA of $5.5-\mathrm{mm}$ diameter variable angle ( $5^{\circ}$ variations) screws $(25-30 \mathrm{~mm})$. For this study, all S-1 screws for the SA group were $30 \mathrm{~mm}$. For the 2 other constructs, a similar interbody spacer (CONTINENTAL, Globus Medical Inc.) was inserted with fixation, either through an anterior tension band plate (PROVIDENCE, Globus Medical Inc.), or bilateral posterior pedicle screws (REVERE, Globus Medical Inc.) in the vertebral body of L-5 and S-1. The ATB constructs used 6.5-mm diameter fixed angle screws inserted to cover $75 \%$ of the anterior body (24-30 mm), while the PPS constructs used $6.5-\mathrm{mm}$ diameter screws inserted to a nominal depth of $75 \%$ of the vertebral body length (45-60 $\mathrm{mm}$ without bicortical purchase at L-5, S-1 screws were all bicortical). All surgical constructs were loaded according to the same load protocol as intact specimens, with compression testing from $20^{\circ}$ to $50^{\circ}$ in increments of $10^{\circ}$.

\section{Testing Apparatus and Protocol}

Each specimen was thawed overnight and mounted on a MTS Bionix frame (MTS) and servohydraulic machine. A custom-built apparatus with an adjustable angle allowed testing at various sacral slopes (Fig. 2). The MTS machine applied a load of $55 \%$ of the average estimated male body weight (200 lbs) to each specimen. A load-control protocol was used to allow specimens to continue their course of motion until the desired 550-N load was met, and the displacement was recorded. All constructs were performed at L5-S1 and in randomized order. Once a construct was inserted, it remained in the specimen until the load protocol for each angle sequence was completed. The last construct on each specimen remained so that load to failure could be tested.

Three specimens per each fixation type ( 9 total) were selected for load to failure testing. Load-deformation curves were collected with a $40^{\circ}$ sacral slope, along with the failure load. Failure was defined as subsidence (or penetration) of the interbody spacer into the cancellous bone noted either by visual or fluoroscopic evidence. Ultimate strength was calculated at the peak of the load displacement curve.

\section{Statistical Analysis}

All statistical analyses were performed using SPSS software (IBM Corp.). Axial stiffness (N/mm) was collected for each specimen across the surgical constructs (intact, SA, PPS, and ATB) and sacral slope angle $\left(20^{\circ}\right.$, $30^{\circ}, 40^{\circ}$, and $50^{\circ}$ ); both were evaluated as repeated-measures variables independent from one another. A 2-way ANOVA with repeated measures and Bonferroni post hoc analysis was used to determine the main effect of both surgical constructs and sacral slope factors on lumbosacral axial stiffness (significance $\mathrm{p} \leq 0.05$ ). Additionally, multiple 1-way ANOVAs with repeated measures and Bonferroni post hoc analyses were performed to assess differences in axial stiffness 1) between surgical constructs at each sacral slope angle and 2) between sacral slope angles for each surgical construct (significance $\mathrm{p} \leq$ 0.05). This allowed analysis of changes of each construct as sacral slope increased, and determination of the stiffest construct at each sacral slope. Lastly, a 1-way independent ANOVA and Bonferroni post hoc analysis was performed to assess differences in load to failure between the construct groups.

\section{Results}

\section{Two-Way ANOVA With Repeated Measures}

The present study investigated the effect of 2 independent factors-testing group (intact, SA, PPS, and ATB) 
and sacral slope $\left(20^{\circ}, 30^{\circ}, 40^{\circ}\right.$, and $\left.50^{\circ}\right)$ - on the axial stiffness of the lumbosacral joint. A 2-way ANOVA with repeated measures (both variables repeated on same specimens) was used to assess the main effect of each factor, independent from the other; this analysis was necessary to determine whether surgical constructs as a whole, sacral slope as a whole, or both affect axial stiffness.

\section{Main Effect of Surgical Constructs on Axial Stiffness}

Mean axial stiffness and standard deviations for each construct are shown in Table 1. Mean axial stiffness across sacral slope at each surgical construct is depicted in Fig. 3. A 2-way repeated-measures ANOVA with assumed sphericity (Mauchly's test, $\mathrm{p}=0.128$ ) determined that while neglecting sacral slope angulation, axial stiffness did not significantly differ between all 3 surgical constructs $(\mathrm{p}=$ 0.218). Post hoc tests using Bonferroni correction revealed that SA demonstrated a lower mean axial compression compared with intact, PPS, and ATB surgical constructs, observed in Fig. 3. However, this decrease was not statistically significant $(\mathrm{p}=0.181, \mathrm{p}=1.00$, and $\mathrm{p}=1.00$, respectively). Significant interaction between surgical construct and sacral slope angle were not found $(\mathrm{p}=0.423)$, as illustrated by the similarity of trends in Fig. 3. Results provide evidence that when pelvic angulation is neglected, the main effect of surgical constructs on lumbosacral stiffness is negligible.

\section{Main Effect of Sacral Slope Angle on Axial Stiffness}

Mean axial stiffness for each sacral slope is shown in Table 2. Mean axial stiffness across surgical constructs at each sacral slope angle is detailed in Fig. 4. The same 2-way repeated-measures ANOVA analysis but with Greenhouse-Geisser correction (Mauchly's test, $\mathrm{p}=$ 0.004) established that while neglecting surgical constructs, axial stiffness varied significantly between sacral slope angles $(\mathrm{p}=0.021)$. Post hoc tests using the Bonferroni correction found the sacral slope angle induced a decrease in axial stiffness from $30^{\circ}$ to $50^{\circ}(312.5 \pm 45.8 \mathrm{~N} /$ $\mathrm{mm}$ vs $201.8 \pm 29.6 \mathrm{~N} / \mathrm{mm}$, respectively), which was statistically significant $(\mathrm{p}=0.017)$. Furthermore, sacral slope angle elicited a reduction in axial stiffness from $40^{\circ}$ to $50^{\circ}$ $(254.2 \pm 36.0 \mathrm{~N} / \mathrm{mm}$ vs $201.8 \pm 29.6 \mathrm{~N} / \mathrm{mm}$, respectively), also statistically significant $(\mathrm{p}=0.021)$. Axial stiffness at $20^{\circ}$ was not found to be statistically different than the other sacral slope angles, in part due to the high standard deviation, shown in Table 2. While the aforementioned overall result did not demonstrate a significant interaction between surgical construct and sacral slope angulation, intact lumbosacral stiffness showed increased sensitivity

TABLE 1. Axial stiffness of intact segment and test constructs*

\begin{tabular}{cc}
\hline Surgical Construct & Mean Axial Stiffness (SD) \\
\hline Intact & $294.5(44.7)$ \\
\hline SA & $244.3(37.3)$ \\
\hline PPS & $301.1(52.7)$ \\
\hline ATB & $274.7(44.4)$ \\
\hline
\end{tabular}

* Units for axial stiffness values are $\mathrm{N} / \mathrm{mm}$.

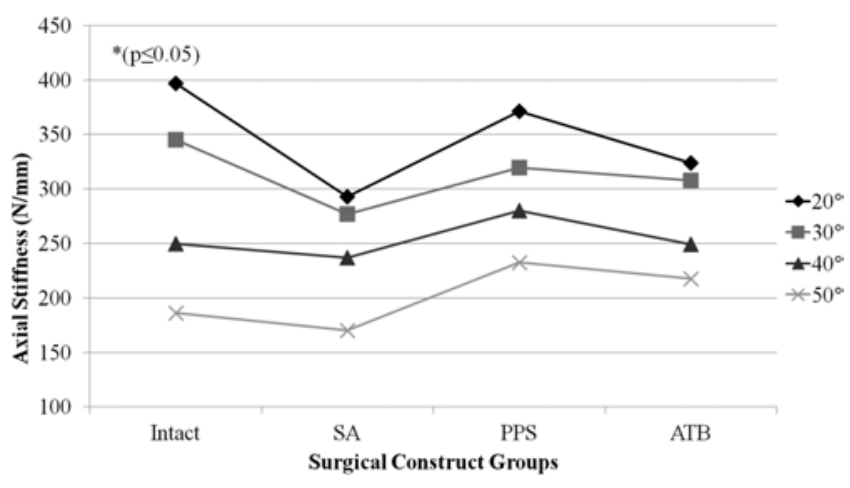

FIG. 3. Graph showing mean axial stiffness across sacral slope angles for each surgical construct.

to sacral slope angles larger than $30^{\circ}$ compared with other constructs, illustrated in Fig. 4. These results demonstrate the main effect of sacral slope angles significantly impacting lumbosacral stiffness. Furthermore, post hoc analysis found that for sacral slopes larger than $40^{\circ}$, axial stiffness of the lumbosacral joint was compromised compared with less angulated pelvic sagittal alignment.

\section{One-Way ANOVA With Repeated Measures}

Whereas the 2-way ANOVA was used to determine the main effect of surgical constructs and sacral slope angles on axial stiffness, multiple 1-way ANOVA with repeated measures and Bonferroni post hoc analyses was performed to determine differences in axial stiffness 1) between surgical constructs at each sacral slope angle and 2) between sacral slope angles for each surgical construct.

\section{Differences Between Surgical Constructs per Sacral Slope Angles}

Lumbosacral stiffness results and statistical significance achieved for all surgical constructs and sacral slope angles are shown in Fig. 5. Previous 2-way ANOVA analysis indicated surgical constructs have a negligible effect on axial stiffness. A 1-way ANOVA with repeated measures corroborated with the previous conclusion at $20^{\circ}, 30^{\circ}, 40^{\circ}$, and $50^{\circ}$ sacral slope angles, with overall $\mathrm{p}$ values of $0.190,0.312,0.771$, and 0.076 , respectively. Post hoc tests using the Bonferroni correction were performed but are not reported, as pairwise comparisons did not detect any significant differences between constructs at any sacral slope angle. In conclusion, at each sacral angulation, the axial stiffness of surgical constructs was not

TABLE 2. Relationship between axial stiffness and sacral slope angle*

\begin{tabular}{cc}
\hline Sacral Slope Angle & Mean Axial Stiffness (SD) \\
\hline $20^{\circ}$ & $346.2(65.6)$ \\
\hline $30^{\circ}$ & $312.5(45.8)$ \\
\hline $40^{\circ}$ & $254.2(36.0)$ \\
\hline $50^{\circ}$ & $201.8(29.6)$ \\
\hline
\end{tabular}

\footnotetext{
* Units for axial stiffness values are $\mathrm{N} / \mathrm{mm}$.
} 


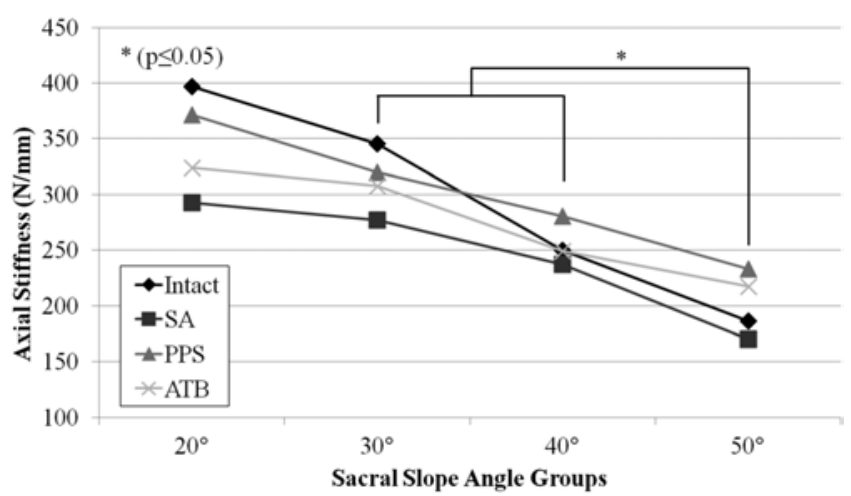

FIG. 4. Graph showing mean axial stiffness across surgical constructs at each sacral slope angle.

found to significantly differ from each other (all comparisons $\mathrm{p} \geq 0.05$ ).

\section{Differences Between Sacral Slope Angles per Constructs.}

Lumbosacral stiffness results for all sacral slopes for each surgical construct and statistical significance achieved are shown in Fig. 6. Previous 2-way ANOVA analysis showed significant decrease in stiffness between both $30^{\circ}$ and $40^{\circ}$, and $30^{\circ}$ and $50^{\circ}$. Multiple 1-way ANOVA with repeated measures and Bonferroni post hoc analyses were performed to assess differences between axial stiffness at sacral slope angles for each construct.

In the intact group, a repeated-measures ANOVA with sphericity assumed (Mauchly's test, $p=0.055$ ) determined that stiffness differed significantly between sacral slope angles $(\mathrm{p}=0.001)$. Post hoc tests using the Bonferroni correction found the sacral slope angle reduced axial stiffness at $50^{\circ}$ compared with $30^{\circ}(186.2 \pm 79.6 \mathrm{~N} / \mathrm{mm}$ vs $345.1 \pm$ $160.2 \mathrm{~N} / \mathrm{mm}$, respectively), a difference that was statistically significant $(\mathrm{p}=0.033)$. Moreover, increasing sacral slope angle from $40^{\circ}$ to $50^{\circ}$ reduced axial stiffness ( 249.8 $\pm 110.9 \mathrm{~N} / \mathrm{mm}$ vs $186.2 \pm 79.6 \mathrm{~N} / \mathrm{mm}$, respectively) with statistical significance $(\mathrm{p}=0.041)$.

Similar trends of significance were observed for the integrated spacer group. A repeated-measures ANOVA with sphericity assumed (Mauchly's test, $p=0.071$ ) determined that stiffness differed significantly between sacral slope angles $(\mathrm{p}=0.000)$. Post hoc tests using the Bonferroni correction found the sacral slope angle induced a decrease in axial stiffness from $30^{\circ}$ to $50^{\circ}(277.2 \pm 113.7 \mathrm{~N} / \mathrm{mm}$ vs $170.3 \pm 74.1 \mathrm{~N} / \mathrm{mm}$, respectively) and from $40^{\circ}$ to $50^{\circ}$ $(237.0 \pm 108.7 \mathrm{~N} / \mathrm{mm}$ vs $170.3 \pm 74.1 \mathrm{~N} / \mathrm{mm}$, respectively), with both reductions showing statistical significance $(\mathrm{p}=$ 0.011 and $p=0.014$, respectively).

Dissimilar to the previous surgical constructs, a repeated-measures ANOVA with Greenhouse-Geisser correction (Mauchly's test, $\mathrm{p}=0.007$ ) determined that in specimens instrumented with PPS, stiffness did not differ statistically significantly between sacral slope angles ( $\mathrm{p}=$ 0.144 ), with pairwise comparison using Bonferroni correction in agreement ( $\mathrm{p} \geq 0.05$ for all comparisons). Lastly, a repeated-measures ANOVA with sphericity assumed (Mauchly's test, $\mathrm{p}=0.051$ ) determined that stiffness did not significantly change between sacral slope angles in the

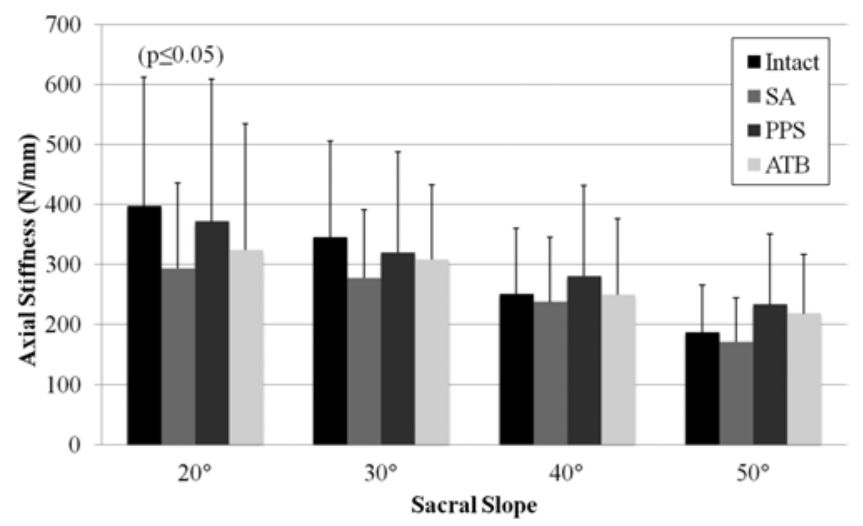

FIG. 5. Bar graph showing L5-S1 means and standard deviations of axial stiffness measurements for sacral slope angles across surgical constructs. No statistically significant difference was found between any constructs at any given sacral slope angle (all comparisons $p \geq 0.05$ ).

ATB construct $(\mathrm{p}=0.081)$. However, post hoc tests using the Bonferroni correction found the sacral slope angle induced a statistically significant $(\mathrm{p}=0.047)$ decrease in axial stiffness from $30^{\circ}$ to $50^{\circ}(307.8 \pm 125.4 \mathrm{~N} / \mathrm{mm}$ vs $217.7 \pm 99.4 \mathrm{~N} / \mathrm{mm}$, respectively).

In review, while a general trend of diminishing axial stiffness was observed as sacral angulation increased, overall results indicate this reduction was only significant in intact and SA constructs. In both of these constructs, significant reduction was found between sacral slope angles of both $30^{\circ}$ and $40^{\circ}$ in comparison with $50^{\circ}$ (p s 0.05). Significant diminution as sacral slope worsened was not found in the overall results for both PPS and ATB constructs $(p \geq 0.05)$; however, post hoc analyses indicated a significant decrease in stiffness between ATB at $30^{\circ}$ and $50^{\circ}(\mathrm{p} \leq 0.05)$.

\section{One-Way Independent ANOVA}

While the original sample size was 9 , due to the destructive nature of biomechanical testing, only final constructs were tested for load to failure with SA, PPS, and ATB ( $n=3$ for each). A 1-way independent ANOVA and Bonferroni post hoc analysis did not show statistically

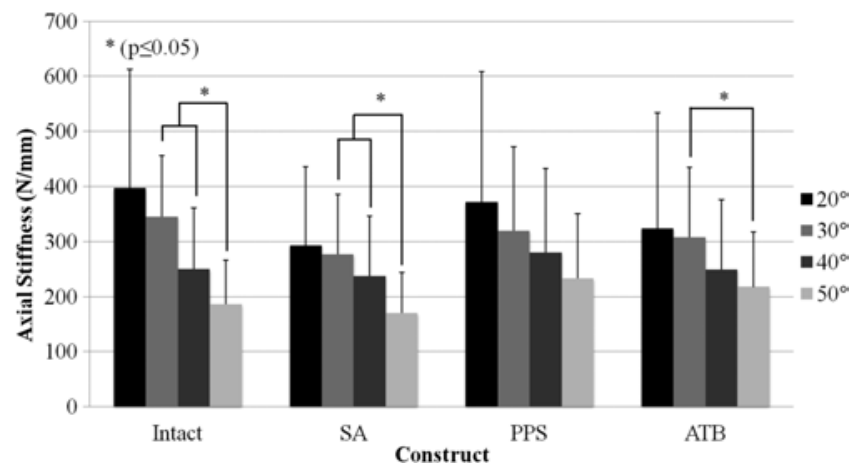

FIG. 6. Bar graph showing L5-S1 means and standard deviations of axial stiffness measurements for surgical constructs across sacral slope angles. Statistically significant differences within surgical construct comparisons are indicated $\left(^{*}\right)(p \leq 0.05)$. 
significant differences between means ( $p \geq 0.05)$, largely due to the small sample size and large standard deviations; results are shown in Fig. 7. The SA construct resulted in the highest load while the ATB construct resulted in the lowest load to failure.

\section{Discussion}

The lumbosacral junction has a significantly higher prevalence of disc degeneration than any other segment of the lower back, except L4-5, often requiring surgical intervention. ${ }^{6}$ Long fusion constructs extending to the sacrum also rely on solid fixation across L5-S1. As such, the complexity of the lumbosacral junction may contribute to reported complications. ${ }^{10,11,35}$ These complications can include screw pullout, implant breakage, or pseudarthrosis, all of which may be related to the lack of stiffness found in this study. ${ }^{10,11}$ These complications may have to do with the high range of motion in this region compared with other lumbar segments. ${ }^{32,41}$ It may also deal with the location and the lordosis of L5-S1.9,17,28,29,39 Due to lordosis, the angle at L5-S1 not only experiences axial forces but also shear forces. This angle influences forces such that greater angles should cause greater shear forces and less axial forces. There seems to be a lack of understanding of these forces at the lumbosacral joint and of how surgical constructs are affected as the angle changes. The present study assessed the influence of this angle in terms of sacral slope and investigated how surgical constructs are affected as sacral slope increases.

In terms of how sacral slope affects each construct, all groups, with the exception of the posterior pedicle screw group, had significant stiffness loss as the angulations approached $50^{\circ}$. There were no significant differences found in stiffness between a sacral slope of $20^{\circ}$ and other tested angles, due high standard deviations. However, there was a noticeable reduction in stiffness at sacral angles equal to or exceeding $40^{\circ}$, potentially due to a redistribution of the applied load. An explanation for this is the transfer of forces from axial force to shear force, causing the spine to translate anteriorly. This illuminates what is seen in spondylolisthesis cases, where high sacral slopes are

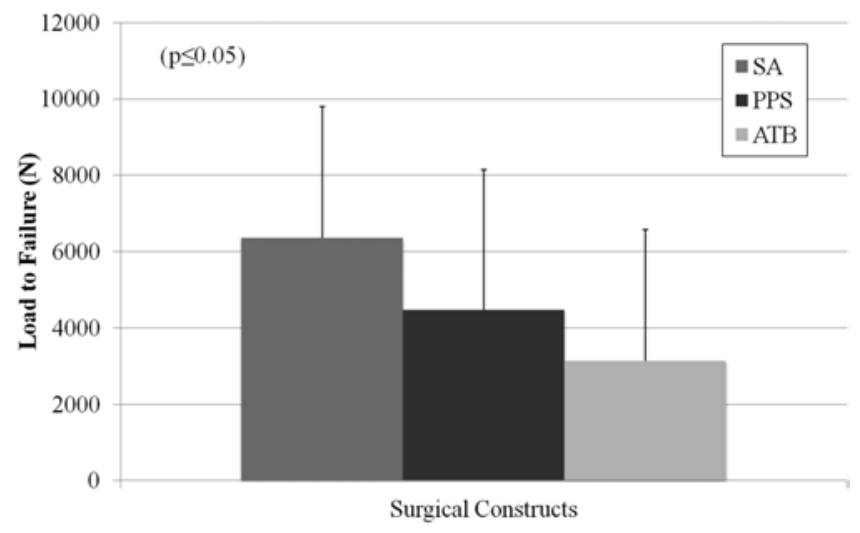

FIG. 7. Bar graph showing load to failure of L5-S1 surgical constructs at a sacral slope of $40^{\circ}$. No statistically significant difference was found between between any constructs (all comparisons $p \geq 0.05$ ). contributing to anterior slip at an above average rate.,.$^{4,18,27}$ Mathematically, as the angles increase beyond $45^{\circ}$, the L5-S1 segment translates more anteriorly then it does compress; however, since L5-S1 does not displace much at low sacral slopes, this trend may occur before $45^{\circ}$. Normal sacral slope angles can be as high as $40^{\circ}$; however, any angle beyond this degree caused significant deformation with the same load applied. This was also observed in the integrated spacer and anterior tension band plate group where the rigidness of the construct was lost due to the shear forces. The stand-alone spacer had the most notable changes, resulting in significant stiffness decreases at $50^{\circ}$ when compared with $30^{\circ}$ and $40^{\circ}$. Unlike other constructs, this construct behaved most similar to intact, and could imply that $40^{\circ}$ sacral slope may be the threshold for standalone spacer usage, which was not the case for anterior band plating only, which failed to show a significant difference from $40^{\circ}$ to $50^{\circ}$. Posterior pedicle screws were the only construct that were not affected statistically by the increase in sacral slope angles, denoting that it was the most consistent of all constructs tested.

Although sacral slope had a significant impact on the intact specimen as well, it was surprising that intact L5-S1 had a high axial stiffness, especially at low sacral slopes. As described previously, the lumbosacral junction has a larger range of motion than any other lumbar segment. ${ }^{32,42}$ It was believed that axial stiffness would be minimal when compared with the constructs. The authors believe that this is due to the angle of L-5 with respect to S-1-which is generally higher than any other lumbar segment. ${ }^{1,19,28,36}$ To achieve this angle, the anatomy is such that the posterior edge of the annulus is shorter in height than the anterior. ${ }^{39}$ The authors believe that this shorter annulus could affect the intact rigidness by resisting compressive loads, which would cause higher stiffness. Additionally, it has been noted that the intact spine does not behave in linear deformation such as metals; as such, comparison with fixation constructs are challenging. ${ }^{37}$ Results from previous studies by Kimura et al. and Li et al. are important because the lack of stiffness increase from intact to the instrumented constructs have also been noted..$^{20,26}$ Kimura et al. reported that compressive differences at L5-S1 loading were not significantly different from the rested height with just natural body weight forces. ${ }^{20}$ This could suggest that L5-S1 has an inherently high stiffness. Although a multilevel study, unlike the single segment study performed here, $\mathrm{Li}$ et al. showed that rigid fixation of L4-5 did not significantly change overall axial stiffness from L3-S1, which also reinforces the findings of this research. ${ }^{26}$ Perhaps the rationale that rigid fixation significantly increases stiffness is due to our knowledge of rotational stiffness. Rotational stiffness of L5-S1 differs from axial stiffness, which has shown significant decreases in range of motion due to stiff constructs, even with complete L-5 spondylectomy. ${ }^{3}$

Comparing all surgical constructs to one another and examining each construct, whether intact, integrated stand-alone spacer, posterior bilateral pedicle screws, or anterior plate, did not yield any differences in stiffness at the same degree of sacral slope. At $20^{\circ}$, all constructs were similar, but the intact spines demonstrated the greatest 
stiffness. There were no statistically significant differences between any groups; however, sacral slopes above $40^{\circ}$ did have a trend shift, and such stiffness significantly fell from $30^{\circ}$ and $40^{\circ}$, as seen in the sacral slope effect comparisons. All constructs lost stiffness, similar to intact, as sacral slope angulations increased; however, a notable trend shift occurred after $30^{\circ}$, causing posterior pedicle screws and anterior tension band plating average stiffness values to be greater at $40^{\circ}$ and $50^{\circ}$ than intact, as seen in Fig. 3. Although it is not a significant increase, this would make these constructs more desirable in situations of high sacral slope.

Conversely, load to failure of each construct resulted in stand-alone integrated spacers being the strongest construct. None of the stand-alone specimens demonstrated endplate subsidence. Instead, specimens failed at the bone screw interface, and 1 of the integrated spacers had a sacral fracture. Previous studies have reported the mean breaking point of bone to be at $6475 \mathrm{~N}$ to $8800 \mathrm{~N} .{ }^{15}$ Hutton et al. also found values as low as $800 \mathrm{~N}$ and as high as nearly $16,000 \mathrm{~N}$ for axial failures of the lumbar spine. ${ }^{15}$ Results from this study fall in line with these fracture rates; however, none of these fractures caused endplate subsidence. Visually, it was observed that at $40^{\circ}$, with high axial loads, the lumbosacral joint resulted in decreased height posteriorly while the anterior portion slightly opened and sheared forward. This effect may elucidate why subsidence is not as prominent for high sacral slopes. As stated previously, high sacral slopes can lead to spondylolisthesis; with corrections, however, the clinical outcomes have been satisfactory as far as fusion and the absence of subsidence is concerned. ${ }^{2,14,16,18}$ Interestingly, although the integrated spacer performed as the least stiff construct, it performed the best in terms of failure. The authors believe this is due to the angulations of the screws through the sacrum and L-5 vertebral body, which were inserted at $30^{\circ}$ to $45^{\circ}$ of the endplate. Unlike pedicle screws or anterior plates, the stand-alone integrated spacer screws are not parallel to the endplate or to the translating plane of L-5. This may lead the screws to simply pull out, whereas stand-alone integrated spacers must actually tear the bone to fail. The discrepancy in load to failure and stiffness at $550 \mathrm{~N}$ is a result of screw/implant migration. At $550 \mathrm{~N}$ stand-alone integrated spacers may have some motion either at the screw to spacer, screw to bone, or spacer migration; however, as loads continue to increase, these smaller motions reach a hard stop and the only method of failure is for the screws to "plow" through the bone. Motion during 550-N compression could have also occurred from the use of variable angle screws. Although variable angle screws provide a more independent trajectory, they may be more susceptible to motion. The anterior plate constructs all used fixed angle screws, which caused less motion during the initial compression. Most importantly, stand-alone integrated spacers have had similar fusion outcomes to that of pedicle screws and anterior plating systems when involving L5-S1. Although the overall stiffness has no statistically significant change with any fixation method, stiffness may not be a proper indicator of clinical outcome when involving high sacral slopes.

Unfortunately, the results of this study prove that 1) there is not a single fixation method that stiffens the lumbosacral significantly, and 2) as sacral slope increases, stiffness is significantly reduced in both intact and fixation constructs. Therefore, it may be clinically relevant to consider both anterior and posterior rigid fixation in cases of high sacral slope to counter the effects of shear force. Such techniques have been used for high-grade spondylolisthesis. ${ }^{22}$ Fusion outcomes using 1 technique have been well reported.24,34 Nemani et al. reported that only $10 \%$ of the 117 patients required revision surgery following a stand-alone treatment of L5-S1. ${ }^{34}$ Therefore, research will be needed to study whether use of more than one construct will improve patient outcomes. In addition, it would be important to assess different implants that have larger lordotic footprints and whether they would increase the stiffness across the constructs at the larger sacral slopes.

This study was a biomechanical investigation, which has inherent limitations. There are no muscles to react to any of the forces applied. The forces applied corresponded to the forces encountered by a 200-lbs individual; however, this may not be accurate for every specimen tested. Specimens, although prepared as quickly as possible, could have degraded from the time of the donor passing until testing, which could affect biomechanical outcomes. Clinically, bone growth and healing that may occur following the operation cannot be tested biomechanically. Lastly, bone mineral density was not obtained for the specimens tested due to cost considerations. Anecdotally, each specimen had adequate bone purchase during screw insertion. Still, each construct was performed on the same specimen, and load to failure constructs were randomized. Furthermore, testing order of the constructs was randomized to avoid bias. The authors believe both protocol procedures adequately counter the effect of varied bone quality.

\section{Conclusions}

At higher sacral slopes, bilateral pedicle screw constructs with spacers were the most stable. Integrated spacers required the highest load for failure, potentially due to the angulated trajectory of the integrated plate screw. High sacral slopes significantly reduced the stiffness of all fixation methods. L5-S1 is naturally a stiff joint in terms of axial compression, and none of the constructs were significantly stiffer than intact. The clinical relevance of this study, therefore, is that surgeons need to consider using more aggressive fixation techniques for a high sacral slope when rigid fixation of the lumbosacral junction is required.

\section{Acknowledgment}

We acknowledge Dr. Melodie Metzger for her previous work with a similar testing apparatus.

\section{References}

1. Baghdadi YM, Larson AN, Dekutoski MB, Cui Q, Sebastian AS, Armitage BM, et al: Sagittal balance and spinopelvic parameters after lateral lumbar interbody fusion for degenerative scoliosis: a case-control study. Spine (Phila Pa 1976) 39:E166-E173, 2014

2. Barrey C, Perrin G, Champain S: Pedicle-screw-based dynamic systems and degenerative lumbar diseases: biome- 
chanical and clinical experiences of dynamic fusion with isobar TTL. ISRN Orthop 2013:183702, 2013

3. Bartanusz V, Muzumdar A, Hussain M, Moldavsky M, Bucklen B, Khalil S: Spinal instrumentation after complete resection of the last lumbar vertebra: an in vitro biomechanical study after L5 spondylectomy. Spine (Phila Pa 1976) 36:1017-1021, 2011

4. Berlemann U, Jeszenszky DJ, Bühler DW, Harms J: The role of lumbar lordosis, vertebral end-plate inclination, disc height, and facet orientation in degenerative spondylolisthesis. J Spinal Disord 12:68-73, 1999

5. Boachie-Adjei O, Dendrinos GK, Ogilvie JW, Bradford DS: Management of adult spinal deformity with combined anterior-posterior arthrodesis and Luque-Galveston instrumentation. J Spinal Disord 4:131-141, 1991

6. Butler D, Trafimow JH, Andersson GB, McNeill TW, Huckman MS: Discs degenerate before facets. Spine (Phila Pa 1976) 15:111-113, 1990

7. Cain CM, Schleicher P, Gerlach R, Pflugmacher R, Scholz M, Kandziora F: A new stand-alone anterior lumbar interbody fusion device: biomechanical comparison with established fixation techniques. Spine (Phila Pa 1976) 30:2631-2636, 2005

8. Carlson GD, Abitbol JJ, Anderson DR, Krag MH, Kostuik JP, Woo SL, et al: Screw fixation in the human sacrum. An in vitro study of the biomechanics of fixation. Spine (Phila Pa 1976) 17 (6 Suppl):S196-S203, 1992

9. Choi S, Lee M, Kwon B: A study on difference and importance of sacral slope and pelvic sacral angle that affect lumbar curvature. Technol Health Care 22:467-472, 2014

10. Edwards CC II, Bridwell KH, Patel A, Rinella AS, Berra A, Lenke LG: Long adult deformity fusions to L5 and the sacrum. A matched cohort analysis. Spine (Phila Pa 1976) 29:1996-2005, 2004

11. Emami A, Deviren V, Berven S, Smith JA, Hu SS, Bradford DS: Outcome and complications of long fusions to the sacrum in adult spine deformity: Luque-Galveston, combined iliac and sacral screws, and sacral fixation. Spine (Phila Pa 1976) 27:776-786, 2002

12. Feng Y, Chen L, Gu Y, Zhang ZM, Yang HL, Tang TS: Influence of the posterior lumbar interbody fusion on the sagittal spino-pelvic parameters in isthmic L5-S1 spondylolisthesis. J Spinal Disord Tech 27:E20-E25, 2014

13. Funao H, Tsuji T, Hosogane N, Watanabe K, Ishii K, Nakamura M, et al: Comparative study of spinopelvic sagittal alignment between patients with and without degenerative spondylolisthesis. Eur Spine J 21:2181-2187, 2012

14. Hsu HT, Yang SS, Chen TY: The correlation between restoration of lumbar lordosis and surgical outcome in the treatment of low-grade lumbar degenerative spondylolisthesis with spinal fusion. J Spinal Disord Tech [epub ahead of print], 2013

15. Hutton WC, Cyron BM, Stott JR: The compressive strength of lumbar vertebrae. J Anat 129:753-758, 1979

16. Kelly AM, Batke JN, Dea N, Hartig DP, Fisher CG, Street JT: Prospective analysis of adverse events in surgical treatment of degenerative spondylolisthesis. Spine J 14:2905-2910, 2014

17. Kim DH, Park JK, Jeong MK: Influences of posterior-located center of gravity on lumbar extension strength, balance, and lumbar lordosis in chronic low back pain. J Back Musculoskelet Rehabil 27:231-237, 2014

18. Kim SY, Maeng DH, Lee SH, Jang JS: Anterior lumbar interbody fusion for lumbosacral junction in steep sacral slope. $\mathbf{J}$ Spinal Disord Tech 21:33-38, 2008

19. Kimura H, Shikata J, Odate S, Soeda T, Yamamura S: Risk factors for cage retropulsion after posterior lumbar interbody fusion: analysis of 1070 cases. Spine (Phila Pa 1976) 37:1164-1169, 2012

20. Kimura S, Steinbach GC, Watenpaugh DE, Hargens AR:
Lumbar spine disc height and curvature responses to an axial load generated by a compression device compatible with magnetic resonance imaging. Spine (Phila Pa 1976) 26:2596-2600, 2001

21. Labelle H, Roussouly P, Berthonnaud E, Dimnet J, O'Brien M: The importance of spino-pelvic balance in L5-s1 developmental spondylolisthesis: a review of pertinent radiologic measurements. Spine (Phila Pa 1976) 30 (6 Suppl):S27S34, 2005

22. Laursen M, Thomsen K, Eiskjaer SP, Hansen ES, Bünger CE: Functional outcome after partial reduction and 360 degree fusion in grade III-V spondylolisthesis in adolescent and adult patients. J Spinal Disord 12:300-306, 1999

23. Lebwohl NH, Cunningham BW, Dmitriev A, Shimamoto N, Gooch L, Devlin V, et al: Biomechanical comparison of lumbosacral fixation techniques in a calf spine model. Spine (Phila Pa 1976) 27:2312-2320, 2002

24. Lee CS, Hwang CJ, Lee DH, Kim YT, Lee HS: Fusion rates of instrumented lumbar spinal arthrodesis according to surgical approach: a systematic review of randomized trials. Clin Orthop Surg 3:39-47, 2011

25. Lee CS, Noh H, Lee DH, Hwang CJ, Kim H, Cho SK: Analysis of sagittal spinal alignment in 181 asymptomatic children. J Spinal Disord Tech 25:E259-E263, 2012

26. Li CD, Sun HL, Lu HZ: Comparison of the effect of posterior lumbar interbody fusion with pedicle screw fixation and interspinous fixation on the stiffness of adjacent segments. Chin Med J (Engl) 126:1732-1737, 2013

27. Lim JK, Kim SM: Difference of sagittal spinopelvic alignments between degenerative spondylolisthesis and isthmic spondylolisthesis. J Korean Neurosurg Soc 53:96-101, 2013

28. MacIntyre NJ, Lorbergs AL, Adachi JD: Inclinometer-based measures of standing posture in older adults with low bone mass are reliable and associated with self-reported, but not performance-based, physical function. Osteoporos Int 25:721-728, 2014

29. Mathios D, Kaloostian PE, Bydon A, Sciubba DM, Wolinsky JP, Gokaslan ZL, et al: A novel method of anterior lumbosacral cage reconstruction. J Neurosurg Spine 20:204-208, 2014

30. Mehta VA, Amin A, Omeis I, Gokaslan ZL, Gottfried ON: Implications of spinopelvic alignment for the spine surgeon. Neurosurgery 70:707-721, 2012

31. Mendoza-Lattes S, Ries Z, Gao Y, Weinstein SL: Proximal junctional kyphosis in adult reconstructive spine surgery results from incomplete restoration of the lumbar lordosis relative to the magnitude of the thoracic kyphosis. Iowa Orthop J 31:199-206, 2011

32. Miyasaka K, Ohmori K, Suzuki K, Inoue H: Radiographic analysis of lumbar motion in relation to lumbosacral stability. Investigation of moderate and maximum motion. Spine (Phila Pa 1976) 25:732-737, 2000

33. Narayan P, Haid RW, Subach BR, Comey CH, Rodts GE: Effect of spinal disease on successful arthrodesis in lumbar pedicle screw fixation. J Neurosurg 97 (3 Suppl):277-280, 2002

34. Nemani VM, Aichmair A, Taher F, Lebl DR, Hughes AP, Sama AA, et al: Rate of revision surgery after stand-alone lateral lumbar interbody fusion for lumbar spinal stenosis. Spine (Phila Pa 1976) 39:E326-E331, 2014

35. Polly DW Jr, Hamill CL, Bridwell KH: Debate: to fuse or not to fuse to the sacrum, the fate of the L5-S1 disc. Spine (Phila Pa 1976) 31 (19 Suppl):S179-S184, 2006

36. Proietti L, Scaramuzzo L, Schiro' GR, Sessa S, Logroscino CA: Complications in lumbar spine surgery: A retrospective analysis. Indian J Orthop 47:340-345, 2013

37. Rostedt M, Ekström L, Broman H, Hansson T: Axial stiffness of human lumbar motion segments, force dependence. J Biomech 31:503-509, 1998 
38. Roussouly P, Pinheiro-Franco JL: Biomechanical analysis of the spino-pelvic organization and adaptation in pathology.

Eur Spine J 20 (Suppl 5):609-618, 2011

39. Vital JM, Boissière L: Total disc replacement. Orthop Traumatol Surg Res 100 (1 Suppl):S1-S14, 2014

40. Vrtovec T, Janssen MM, Likar B, Castelein RM, Viergever MA, Pernuš F: A review of methods for evaluating the quantitative parameters of sagittal pelvic alignment. Spine J 12:433-446, 2012

41. Wong KW, Leong JC, Chan MK, Luk KD, Lu WW: The flexion-extension profile of lumbar spine in 100 healthy volunteers. Spine (Phila Pa 1976) 29:1636-1641, 2004

42. Zhang JD, Poffyn B, Sys G, Uyttendaele D: Are stand-alone cages sufficient for anterior lumbar interbody fusion? Orthop Surg 4:11-14, 2012

\section{Disclosure}

Funding from the Haid Award through the AANS/CNS Section on Disorders of the Spine and Peripheral Nerves, sponsored by Globus, was received in support of this work. The authors report that the study was performed in the biomechanical laboratory within Globus Medical, Inc., using their testing apparatus. Mr. Hussain, Mr. Harris, Mr. Hao, Mr. Phillips, and Dr. Bucklen report being employees of Globus Medical, Inc. Dr. Kim reports being a consultant for DePuy Synthes and receiving support for non-study-related clinical or research effort from Medtronic.

\section{Author Contributions}

Conception and design: Drazin, Bucklen. Acquisition of data: Phillips. Analysis and interpretation of data: Harris, Hao. Drafting the article: Drazin, Hussain. Critically revising the article: Kim, Johnson. Reviewed submitted version of manuscript: Drazin. Approved the final version of the manuscript on behalf of all authors: Drazin. Statistical analysis: Harris.

\section{Supplemental Information}

Previous Presentation

Portions of this paper were presented in 2014 at the 30th Annual Meeting of the AANS/CNS Section on Disorders of the Spine and Peripheral Nerves (March 5-8, Orlando, Florida).

\section{Correspondence}

Doniel Drazin, Department of Neurosurgery, Cedars-Sinai Medical Center, The Spine Center, 444 S. San Vicente Blvd., Mark Goodson Bldg., Ste. 800, Los Angeles, CA 90048. email: doniel. drazin@cshs.org. 